\title{
A Staged Operation as a Surgical Strategy for a Patient with Type VI Isolated Superior Mesenteric Artery Dissection
}

\author{
Ryosuke Nishi ${ }^{a}$ b Yasuhiko Fujita ${ }^{a}$ Teruyoshi Amagai ${ }^{a, c}$ \\ aDepartment of Medicine, Tokunoshima Tokushukai General Hospital, Kagoshima, Japan; \\ ${ }^{b}$ Department of Neurosurgery, Uji Tokushukai General Hospital, Kyoto, Japan; 'Department \\ of Clinical Engineering, Faculty of Health Care Sciences, Jikei University of Health Care \\ Sciences, Osaka, Japan
}

\section{Keywords}

Superior mesenteric artery dissection · Staged operation · Curved multiplanar reconstruction

\begin{abstract}
An isolated superior mesenteric artery (SMA) dissection (ISMAD) is extremely rare among visceral artery dissections. Its diagnosis is made by abdominal contrast CT scan which shows SMA occlusion partially or completely. The ISMAD is classified into 6 types: type I-V has partial occlusion and treated medically using antiplatelets or anticoagulants. On the other hand, type VI has complete occlusion and must be treated by urgent surgical operation. We present a 67-year-old female who presented with sudden onset abdominal pain and melena. An urgent contrast CT revealed type VI ISMAD. She underwent 3 staged operations as follows: (1) first, as laparotomy showed pale color in almost the extensive length of the small intestine, arterial bypassing of SMA was undertaken using SMA to the right common iliac artery bypass; (2) as the second-look operation on the next day, the terminal ileum was resected, and the remaining small intestine was able to be preserved. However, when the abdomen was tried to be closed, systemic blood pressure decreased to pre-shock condition, so the abdominal wall was closed at skin level with silastic sheet. (3) As the third-look operation on the 7th day, ileostomy was created, and the abdominal wall was safely closed. The postoperative course was uneventful. This case study shows that SMA grafting and staged operations might be an option to preserve the length of the small intestine when ISMAD is diagnosed as type VI.

(C) 2021 The Author(s).

Published by S. Karger AG, Basel
\end{abstract}




\section{Case Reports in Gastroenterology}

\begin{tabular}{l|l}
\hline Case Rep Gastroenterol 2021;15:715-719 \\
\hline DOI: 10.1159/000518018 & $\begin{array}{l}\text { @ 2021 The Author(s). Published by S. Karger AG, Basel } \\
\text { www.karger.com/crg }\end{array}$ \\
\hline
\end{tabular}

Nishi et al.: Staged Operation for a Patient with Type VI Superior Mesenteric Artery Dissection

\section{Introduction}

An isolated superior mesenteric artery (SMA) dissection (ISMAD) is extremely rare among the visceral artery dissections. It was first reported in 1947 by Bauersfeld [1]. While dissection lesion involves SMA and its branches with aortic involvement, an ISMAD occurs without an aortic lesion. Its incidence is estimated to be $0.06 \%$ in postmortem studies [2, 3]. ISMAD is reported more frequent in males in their 50 s. It is classified into 6 subtypes: type I, patent type with an entry and re-entry; type II, "cul-de-sac" type without re-entry; type III, with a thrombosed false lumen with ulcer-like projection; type IV, with a completely thrombosed false lumen without ulcer-like projection; type $\mathrm{V}$, accompanied by dissection and stenosis of the SMA; and type VI, with partial or complete occlusion [4]. We experienced consecutively 3 cases with ISMAD in the last 4 years. Among them, the last one was a female with an ISMAD of type VI treated with SMA bypass and ileotomy followed by management by home parenteral nutrition for 6 months. We compared this surgical case with the other 2 experienced cases treated medically to differentiate the surgical indication of the case with ISMAD from medical cases using classification of their subtypes.

\section{Case}

A 67-year-old female presented with sudden onset abdominal pain and melena. She had no medical history. On physical examination, she showed muscle rebound tenderness in the epigastric region that has been lasting for the previous $3 \mathrm{~h}$ and getting more painful, whereas her Glasgow Coma Scale was E4V4M5. The urgent abdominal contrast CT scan showed complete occlusion of the SMA (Fig. 1, 2) and was diagnosed as ISMAD. With this diagnosis, she underwent urgent operations, 3 staged operations, as follows: (1) first, as laparotomy showed pale color in almost the extensive length of the small intestine, arterial bypassing of SMA was undertaken using SMA to the right common iliac artery bypass; (2) second, as the second-look operation on the next day, $150 \mathrm{~cm}$ of the terminal ileum was resected, and the remaining small intestine was able to be preserved. However, when the abdomen was tried to be closed, systemic blood pressure decreased to pre-shock condition, so the abdominal wall was closed at skin level with silastic sheet to prevent abdominal compartment syndrome. (3) Third, as the third-look operation on the 7th day, ileostomy was created, and the abdominal wall was safely closed. The postoperative course was uneventful.

\section{Discussion}

The modified classification of ISMAD and its clinical usefulness: when patients present with complaints of the triad (severe abdominal pain with minimal findings on examination, bowel emptying such as diarrhea, and the presence of a source of embolus, most often arterial fibrillation) [5], diagnosis of ISMAD must be made with urgent ultrasound sonography and contrasted CT imaging modalities to visualize SMA patency. The morphology of ISMAD has been classified into 6 subtypes (see online suppl. Fig. 1; for all online suppl. material, see www.karger.com/doi/10.1159/000518018) [4]. In our 3 experienced cases, the presented case was diagnosed as type VI. Type VI was added to the original classification [6]. Among this modified classification, type VI seems uniquein its necessity of surgical management discussed in the next section. The differential diagnosis of ISMAD including chronic SMA obstruction, especially median arcuate ligament syndrome, must be considered [7].

\section{Karger'"}




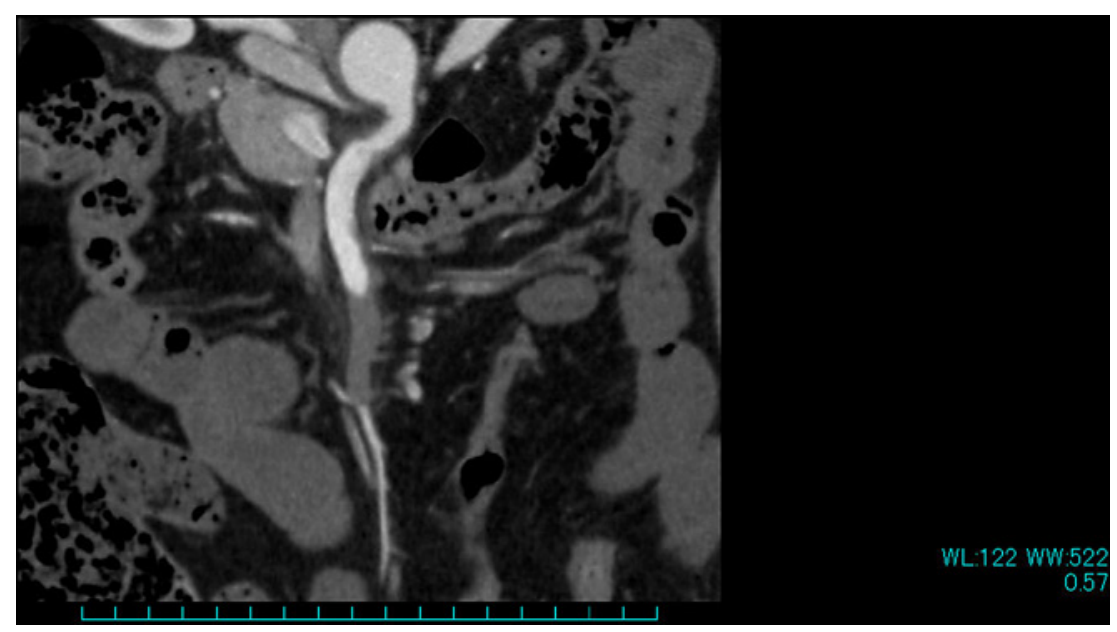

Fig. 1. The left oblique view of contrasted CT reconstructed by curved multiplanar reconstruction. This imaging view shows an abrupt obstruction of the superior mesenteric artery.

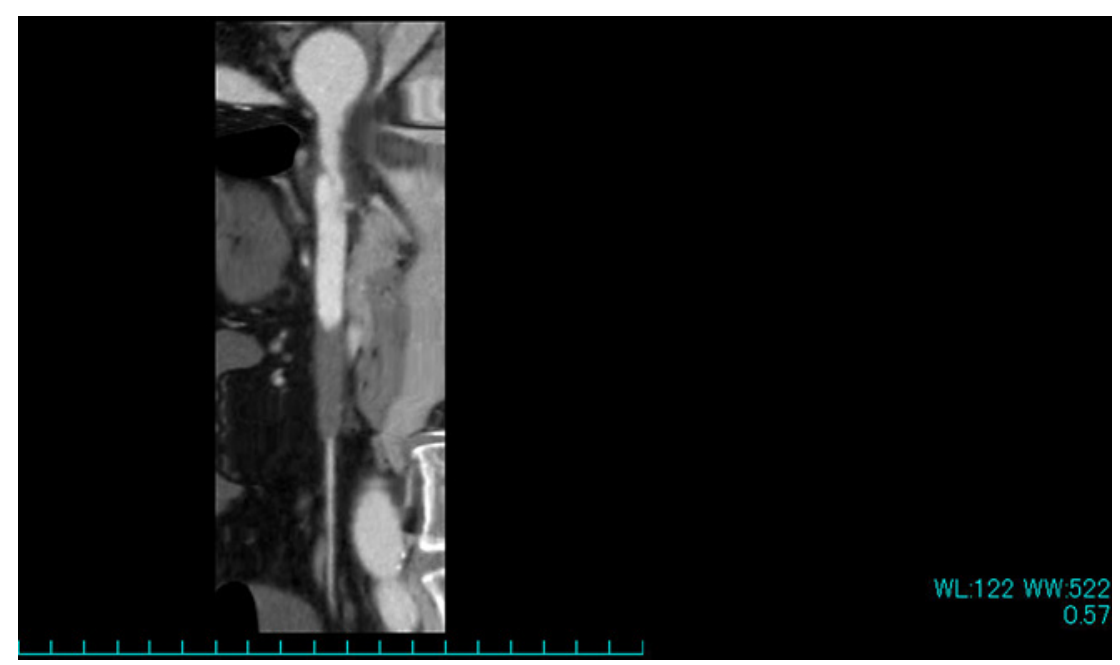

Fig. 2. The front view of contrasted CT reconstructed by curved multiplanar reconstruction. This imaging view also shows an abrupt obstruction of the superior mesenteric artery.

Treatment strategy for patients with ISMAD - medical versus surgical: 2 of our 3 experienced cases were diagnosed type III and treated medically. The type III ISMAD has a true lumen with a thrombosed false lumen. Contrary to type III and the other 4 types, type VI has completely obstructed true and false lumens [4]. When the type of ISMAD is identified, for patients with type $I \sim V$, medical treatments include antiplatelet agents for arterial thrombosis and anticoagulants to prevent clot and emboli formation. In contrary, when type VI is diagnosed, surgical treatment must be considered. The authors of the modified classification of this entity also showed that 2 out of 14 cases were diagnosed as type VI and underwent surgical treatments: stenting or ilio-iliac artery bypassing. The remaining 12 cases were treated conservatively [4]. In general, conservative blood control management is recommended as the first choice because of the self-limited clinical course [8]. However, a case with type VI must not be treated conservatively but surgically, including arterial intervention or staged operations, when massive intestinal resection 


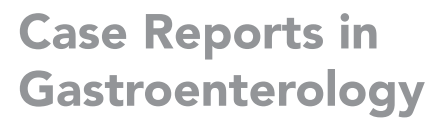

Nishi et al.: Staged Operation for a Patient with Type VI Superior Mesenteric Artery Dissection

and short bowel syndrome must be avoided [9]. Though in the article studying treatment strategies for patients with ISMAD [10] staged operation including observational secondlook operation was not proposed, we emphasize the clinical importance of a staged procedure as a surgical strategy to prevent massive intestinal removal. From these considerations, we propose that when a patient presents with any of the triad symptoms, urgent ultrasound sonography and contrasted CT must be carried out to classify the type of the ISMAD, and urgent surgical treatment must be selected to preserve the small intestine when the type of ISMAD is type VI.

\section{Conclusion}

The presented case with type VI ISMAD was treated with SMA bypassing followed by staged operations to spare the length of small intestinal resection. These urgent surgical treatments including staged operation seem helpful and preventive to massive small intestinal resection when ISMAD is diagnosed as type VI.

\section{Acknowledgment}

The authors sincerely thank Dr. Kazufumi Miyagi, the attending surgeon working in the Department of Surgery, Chubu Tokushukai Hospital, Okinawa, Japan, for his extensively experienced surgical supports for this case.

\section{Statement of Ethics}

This case was approved by the Ethics Committee of Tokunoshima Tokushukai General Hospital (No. 20-4), and written informed consent was obtained from the patient's son for publication of this case report and any accompanying images. The reason why the written informed consent was not obtained from the patient was because of her impaired cognitive function.

\section{Conflict of Interest Statement}

The authors declare that they have no conflicts of interest.

\section{Funding Sources}

The authors did not receive any funding.

\section{Author Contributions}

The data of the reported cases were collected by Dr. Y.N. and T.A. The article was written by Dr. Y.N. and reviewed by Dr. Y.F. and T.A. The final version of the written article was reviewed by Drs. Y.N. and Y.F. and T.A.

\section{Karger'k}


Nishi et al.: Staged Operation for a Patient with Type VI Superior Mesenteric Artery Dissection

\section{Data Availability Statement}

All data generated or analyzed during this study are included in this article and its online suppl. material files. Further enquiries can be directed to the corresponding author.

\section{References}

1 Bauersfeld SR. Dissecting aneurysm of the aorta; a presentation of 15 cases and a review of the recent literature. Ann Intern Med. 1947;26(6):873-89.

2 Park YJ, Park KB, Kim D-I, Do YS, Kim D-K, Kim Y-W. Natural history of spontaneous isolated superior mesenteric artery dissection derived from follow-up after conservative treatment. J Vasc Surg. 2011;54(6):1727-33.

3 Baldino G, Mortola P, Cambiaso M, Valdata A, Gori A. Endovascular treatment with flow-diverting stents of symptomatic superior mesenteric artery dissection aneurysm. J Vas Surg Cases Innov Tech. 2017;61:186788.

4 Satokawa H, Takase S, Seto Y, Yokoyama H, Gotoh M, Kogure M, et al. Management strategy of isolated spontaneous dissection of the superior mesenteric artery. Ann Vasc Dis. 2014;7(3):232-8.

5 Björck M, Koelemay M, Acosta S, Concalves B, Kölbel T, Kolkman IJ, et al. Editor's choice-management of the diseases of mesenteric arteries and veins. Clinical practice guidelines of the European society of vascular surgery (ESVS). Eur J Vasc Endovasc Surg. 2017;53:460-510.

6 Sakamoto I, Ogawa Y, Sueyoshi E, Fukui K, Murakami T, Uetani M. Imaging appearances and management of isolated spontaneous dissection of the superior mesenteric artery. Eur J Radiol. 2007;64(1):103-10.

7 Terlouw LG, Moelker A, Abrahamsen J, Acosta S, Bakker OJ, Baumgartner I, et al. European guidelines on chronic mesenteric ischaemia joint united European gastroenterology, European association for gastroenterology, endoscopy and nutrition, European society of gastrointestinal and abdominal radiology, Netherlands association of hepatogastroenterologists, hellenic society of gastroenterology, cardiovascular and interventional radiological society of Europe, and Dutch mesenteric ischemia study group clinical guidelines on the diagnosis and treatment of patients with chronic mesenteric ischaemia. Uni Eur Gastroenterol J. 2020;8(4): 371-95.

8 Barker SG, Burnand KG. Retrograde iliac artery dissection in Marfan's syndrome. A case report. J Cardiovasc Surg. 1989;30(6):953-4.

9 Wee-Thong N, Peter Ashley Robless. Short bowel syndrome after endovascular recanalization of superior mesenteric artery embolic occlusion: a rare event. J Vasc Interv Radiol. 2012;23(12):1709-11.

10 Jia Z, Tu J, Jiang G. The classification and management strategy of spontaneous isolated superior mesenteric artery dissection. Korean Circ J. 2017;47(4):425-31. 